\title{
Universality of nucleon-nucleon short-range correlations: two-nucleon momentum distributions in few-body systems.
}

\author{
M. Alvioli, ${ }^{1}$ C. Ciofi degli Atti, ${ }^{2}$ L. P. Kaptari,, , C. B. Mezzetti, ${ }^{2}$ H. Morita, ${ }^{3}$ and S. Scopetta ${ }^{2}$ \\ ${ }^{1} E C T^{\star}$, European Center for Theoretical Studies in Nuclear Physics and Related Areas, \\ Strada delle Tabarelle 286, I-38123 Villazzano (TN) Italy \\ ${ }^{2}$ Department of Physics, University of Perugia and \\ Istituto Nazionale di Fisica Nucleare, Sezione di Perugia \\ Via A. Pascoli, I-06123, Italy \\ ${ }^{3}$ Sapporo Gakuin University, Bunkyo-dai 11, Ebetsu 069-8555, Hokkaido, Japan
}

(Dated: January 25, 2018)

\begin{abstract}
Using realistic wave functions, the proton-neutron and proton-proton momentum distributions in ${ }^{3} \mathrm{He}$ and ${ }^{4} \mathrm{He}$ are calculated as a function of the relative, $k_{r e l}$, and center of mass, $K_{C M}$, momenta, and the angle between them. For large values of $k_{r e l} \gtrsim 2 \mathrm{fm}^{-1}$ and small values of $K_{C M} \lesssim$ $1.0 \mathrm{fm}^{-1}$, both distributions are angle independent and decrease with increasing $K_{C M}$, with the $p n$ distribution factorizing into the deuteron momentum distribution times a rapidly decreasing function of $K_{C M}$, in agreement with the two-nucleon $(2 \mathrm{~N})$ short range correlation (SRC) picture. When $K_{C M}$ and $k_{r e l}$ are both large, the distributions exhibit a strong angle dependence, which is evidence of three-nucleon (3N) SRC. The predicted center-of-mass and angular dependence of $2 \mathrm{~N}$ and $3 \mathrm{~N}$ SRC should be observable in two-nucleon knock-out processes $A\left(e, e^{\prime} p N\right) X$.
\end{abstract}

PACS numbers: 21.30.Fe, 21.60.-n, 24.10.Cn, 25.30.-c

Realistic many-body calculations (see e.g. [1]-[3]) show that a mean field approach, though describing very successfully many properties of nuclei, breaks down when the relative distance $r \equiv\left|\boldsymbol{r}_{1}-\boldsymbol{r}_{2}\right|$ between two generic nucleons "1" and " 2 " is of the order of $r \lesssim 1.3-1.5 \mathrm{fm}$. In this region nucleon-nucleon $(\mathrm{NN})$ motion exhibits SRC, arising from the interplay between the short range repulsion and the intermediate range tensor attraction of the NN potential. As a result of such an interplay, the two-nucleon density distribution strongly deviates from the mean field distribution, in that whereas the latter has a maximum value at zero separation, the former almost vanishes at $r=0$, increases sharply with increasing separation, overshoots at $r \gtrsim 1.3-1.5 \mathrm{fm}$ the mean field density, and coincides with it at larger separations. The detailed structure of SRC depends upon the spin-isospin state of the NN pair, as well as upon the value of the pair center-of-mass $(\mathrm{CM})$ coordinate $\boldsymbol{R}=\left(\boldsymbol{r}_{1}+\boldsymbol{r}_{2}\right) / 2$. The study of SRC represents one of the main challenges of nowadays nuclear physics, since the detailed theoretical and experimental knowledge of the short range structure of nuclei, could provide decisive answers to longstanding fundamental questions, such as the formation and structure of cold dense nuclear matter, the origin of the EMC effect, the role of quark-gluon degrees of freedom in nuclei (see e.g. [4]). SRC generate high momentum components, which are lacking in a mean field approach, and give rise to peculiar configurations of the nuclear wave function in momentum space [5]. In particular, if nucleons "1" and "2" become strongly correlated at short distances, the local configuration (in the nucleus $\mathrm{CM}$ frame) characterized by $\boldsymbol{k}_{2} \simeq-\boldsymbol{k}_{1}, \boldsymbol{K}_{A-2}=\sum_{i=3}^{A} \boldsymbol{k}_{i} \simeq 0$, dominates over the average mean field configuration $\sum_{i=2}^{A} \boldsymbol{k}_{i} \simeq-\boldsymbol{k}_{1}$, which is the configuration when the high momentum nucleon is balanced by all of the remaining $A-1$ nucleons. Thus, if a correlated nucleon with momentum $\boldsymbol{k}_{1}$ acquires a momentum $\boldsymbol{q}$ from an external probe and it is removed from the nucleus and detected with momentum $\boldsymbol{p}=\boldsymbol{k}_{1}+\boldsymbol{q}$, the partner nucleon should be emitted with momentum $\boldsymbol{k}_{2} \simeq-\boldsymbol{k}_{1}=\boldsymbol{q}-\boldsymbol{p} \equiv \boldsymbol{p}_{\text {miss }}$. Such a qualitative picture is strictly valid only if the CM momentum of the correlated pair was zero before nucleon removal and, moreover, if the two correlated nucleons leave the nucleus without interacting between themselves and with the nucleus $(A-2)$. Nonetheless, recent experimental data on nucleon knockout from carbon using protons [6], 7] and electron [8] projectiles, have shown that the removal of a proton from the nucleus in the range of $0.275 \lesssim\left|\boldsymbol{p}_{\text {miss }}\right| \lesssim 0.550 \mathrm{GeV} / \mathrm{c}$ is almost always accompanied by the emission of a neutron carrying momentum equal to $\boldsymbol{p}_{m i s s}$, with a momentum spread in agreement with a Gaussian motion of the CM of the correlated pair in the nucleus, as predicted long ago in Ref. 9]. Whereas experiments demonstrating the presence of SRC in nuclei and their basic mechanism have eventually been performed, detailed information through the periodic Table of their isospin, angular and CM dependencies is still to come. A partial relevant progress has however already been done by demonstrating [3, 10], in qualitative agreement with the experimental data on ${ }^{12} \mathrm{C}$, that the strong correlations induced by the tensor force lead to large differences in the $p p$ and $p n$ distributions at moderate values of the relative momentum of the pair. Such a result has been confirmed in a recent thorough analysis [11] of the relative (integrated over the pair CM 
variables $\mathbf{R}$ and $\mathbf{K}_{C M}=\mathbf{k}_{1}+\mathbf{k}_{2}$ ) two-body densities and momentum distributions, and their detailed dependence upon the spin-isospin states. As for the angular and CM dependencies of SRC, in Ref. [3, 10] the focus was on the two-body momentum distributions integrated either over the $\mathrm{CM}$ or the relative momenta, whereas in Ref. 12] the CM dependence of the relative momentum distributions of ${ }^{3} \mathrm{He}$ and ${ }^{4} \mathrm{He}$ has been investigated in a particular angular configuration, namely when $\boldsymbol{K}_{C M}$ and $\boldsymbol{k}_{r e l} \equiv\left(\mathbf{k}_{1}-\mathbf{k}_{2}\right) / 2$ are parallel. In this Letter the results of calculations for arbitrary mutual orientations of
$\boldsymbol{K}_{C M}$ and $\boldsymbol{k}_{r e l}$, are presented and several universal features of SRC will be demonstrated. Our calculations were performed with nuclear wave functions [13, 14] obtained from the solution of the Schrödinger equation containing realistic NN interactions, namely the $A V 18$ [15] and $A V 8^{\prime}$ [16] interactions. We will compare our results with a preliminary analysis of data on ${ }^{3} \mathrm{He}$ from the CEBAF large acceptance spectrometer (CLAS) collaboration at JLab [17]. The summed over spin and isospin two body momentum distributions of a nucleon-nucleon pair is defined as follows

$$
n^{N N}\left(\boldsymbol{k}_{1}, \boldsymbol{k}_{2}\right)=\frac{1}{(2 \pi)^{6}} \int d \boldsymbol{r}_{\mathbf{1}} d \boldsymbol{r}_{\mathbf{2}} d \boldsymbol{r}_{1}^{\prime} d \boldsymbol{r}_{\mathbf{2}}{ }^{i} e^{i \boldsymbol{k}_{1} \cdot\left(\boldsymbol{r}_{1}-\boldsymbol{r}_{1}^{\prime}\right)} e^{i \boldsymbol{k}_{2} \cdot\left(\boldsymbol{r}_{2}-\boldsymbol{r}_{2}^{\prime}\right)} \rho_{N N}^{(2)}\left(\boldsymbol{r}_{1}, \boldsymbol{r}_{2} ; \boldsymbol{r}_{1}^{\prime}, \boldsymbol{r}_{2}^{\prime}\right)
$$

with

$$
\rho_{N N}^{(2)}\left(\boldsymbol{r}_{1}, \boldsymbol{r}_{2} ; \boldsymbol{r}_{1}^{\prime}, \boldsymbol{r}_{2}^{\prime}\right)=\frac{1}{2 J+1} \sum_{M} \int \psi_{J M}^{*}\left(\boldsymbol{r}_{1}, \boldsymbol{r}_{2}, \boldsymbol{r}_{3} \ldots, \boldsymbol{r}_{A}\right) \psi_{J M}\left(\boldsymbol{r}_{1}^{\prime}, \boldsymbol{r}_{2}^{\prime}, \boldsymbol{r}_{3}, \ldots, \boldsymbol{r}_{A}\right) \delta\left(\sum_{i=1}^{A} \boldsymbol{r}_{i}\right) \prod_{i=3}^{A} d \boldsymbol{r}_{i}
$$

being the two-body non diagonal density matrix. In Eq. (2) $\psi_{J M}\left(\boldsymbol{r}_{1}, \boldsymbol{r}_{2}, \boldsymbol{r}_{3} \ldots, \boldsymbol{r}_{A}\right)$ is the wave function of the nucleus in the ground state with the total angular mo- mentum $J$ and its projection $M$. The two-nucleon momentum distribution can then be defined as follows

$n^{N N}\left(\boldsymbol{k}_{r e l}, \boldsymbol{K}_{C M}\right)=n^{N N}\left(k_{r e l}, K_{C M}, \Theta\right)=\frac{1}{(2 \pi)^{6}} \int d \boldsymbol{r} d \boldsymbol{R} d \boldsymbol{r}^{\prime} d \boldsymbol{R}^{\prime} e^{i \boldsymbol{K}_{C M} \cdot\left(\boldsymbol{R}-\boldsymbol{R}^{\prime}\right)} e^{i \boldsymbol{k}_{r e l} \cdot\left(\boldsymbol{r}-\boldsymbol{r}^{\prime}\right)} \rho_{N N}^{(2)}\left(\boldsymbol{r}, \boldsymbol{R} ; \boldsymbol{r}^{\prime}, \boldsymbol{R}^{\prime}\right),(3)$

where $\left|\boldsymbol{k}_{r e l}\right| \equiv k_{r e l},\left|\boldsymbol{K}_{C M}\right| \equiv K_{C M}$ and $\Theta$ is the angle between $\boldsymbol{k}_{r e l}$ and $\boldsymbol{K}_{C M}$. In what follows the momentum distributions are normalized to unity. Given the formula above, the momentum distribution integrated over the CM coordinate, $n_{r e l}^{N N}\left(k_{r e l}\right)$, and the one integrated over the relative momentum, $n_{C M}^{N N}\left(K_{C M}\right)$, can be obtained, but a more important property, considered in this Letter, is the dependence of the two-body momentum distribution upon the relative momentum $k_{r e l}$ for fixed values of the CM momentum $K_{C M}$ and the angle $\Theta$. In our calculations we used, for ${ }^{3} \mathrm{He}$, the nuclear wave function obtained within the approach from Ref. [13] and corresponding to the $A V 18$ interaction [15] and, for ${ }^{4} \mathrm{He}$ the wave functions of Ref. 14] corresponding to the $A V 8^{\prime}$ in- teraction [16]. Before discussing our results, let us stress that the independence of the two-nucleon momentum distributions upon the angle $\Theta$, is evidence of the factorization of the distributions in the variables $k_{r e l}$ and $K_{C M}$, i.e. $n^{N N}\left(k_{r e l}, K_{C M}, \Theta\right) \simeq n_{r e l}^{N N}\left(k_{r e l}\right) n_{C M}^{N N}\left(K_{C M}\right)$ [18, 19]. The $p n$ and $p p$ relative momentum distributions, plotted vs. $k_{r e l}$ in correspondence of several values of $K_{C M}$ and two angular configurations are shown in Figs. 1 and 22 the ratio $R^{p n}=n^{p n}\left(k_{r e l}, 0\right) / n_{D}\left(k_{r e l}\right)$ for back-toback nucleons $\left(n^{p n}\left(k_{r e l}, 0\right) \equiv n^{p n}\left(k_{r e l}, K_{C M}=0\right)\right)$ is presented in Fig. 3, whereas the $p N \mathrm{CM}$ momentum distributions $n_{C M}^{p N}\left(K_{C M}\right)=\int n^{p N}\left(\boldsymbol{k}_{r e l}, \boldsymbol{K}_{C M}\right) d \boldsymbol{k}_{r e l}$ are given in Fig. [4 finally, in Fig. 5 the ratio $R_{p p / p n}$ of the correlated $p p$ to $p n$ pairs, extracted from the ${ }^{3} \mathrm{He}\left(e, e^{\prime} p p\right) n$ 


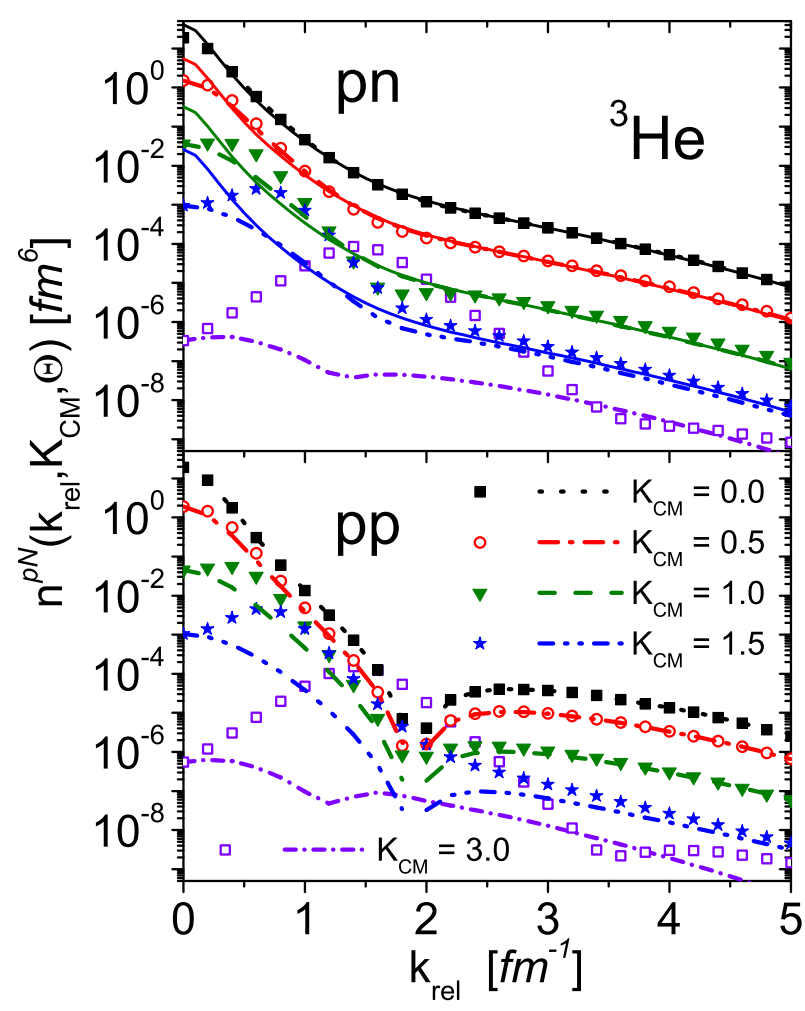

FIG. 1: The two-body momentum distributions of $p n$ and $p p$ pairs in ${ }^{3} \mathrm{He}$ normalized to unity, vs. the relative momentum $k_{r e l}$, for fixed values of the CM momentum $K_{C M}$ and two orientations of them: $\mathbf{k}_{\text {rel }} \| \mathbf{K}_{C M}$ (broken curves) and $\mathbf{k}_{r e l} \perp \mathbf{K}_{C M}$ (symbols). The continuous curves for the $p n$ pair represents the deuteron momentum distribution rescaled by the CM momentum distribution $n_{C M}^{p n}\left(K_{C M}\right)=$ $\int n^{p n}\left(\boldsymbol{k}_{r e l}, \boldsymbol{K}_{C M}\right) d \boldsymbol{k}_{r e l}$ (see text and Fig. 4). ${ }^{3} \mathrm{He}$ wave function from Ref. [13] and $A V 18$ interaction [15].

process [17], is shown.

The main features of our results can be summarized as follows: (i) at $K_{C M}=0$ the results of Ref. [10] are reproduced, namely at small values of $k_{r e l}$ the $p n$ and $p p$ momentum distributions do not appreciably differ, with their ratio being closer to the ratio of the $p n$ to $p p$ pairs, whereas at $1.0 \lesssim k_{\text {rel }} \lesssim 4.0 \mathrm{fm}^{-1}$ the dominant role of tensor correlations makes the $p n$ distributions much larger than $p p$ distribution, with the node exhibited by the latter filled up by the $D$ wave in the $p n$ two-body density; (ii) $n^{N N}\left(k_{r e l}, K_{C M}, \Theta\right)$, plotted vs. $k_{r e l}$, decreases with increasing values of $K_{C M}$; (iii) starting from a given value of $k_{r e l}$, which for $K_{C M}=0$ is $k_{r e l} \simeq 1.5 \mathrm{fm}^{-1}$, and increases with increasing $K_{C M}$, the $p n$ distribution changes its slope and becomes close to the deuteron distribution; (iv) in the region $\left(k_{r e l} \gtrsim 2 \mathrm{fm}^{-1}, K_{C M} \lesssim 1 \mathrm{fm}^{-1}\right)$, $n^{N N}$ becomes $\Theta$-independent, 25], which means that $n^{N N}\left(k_{r e l}, K_{C M}, \Theta\right) \simeq n_{r e l}^{N N}\left(k_{r e l}\right) n_{C M}^{N N}\left(K_{C M}\right) ;$ for $p n$

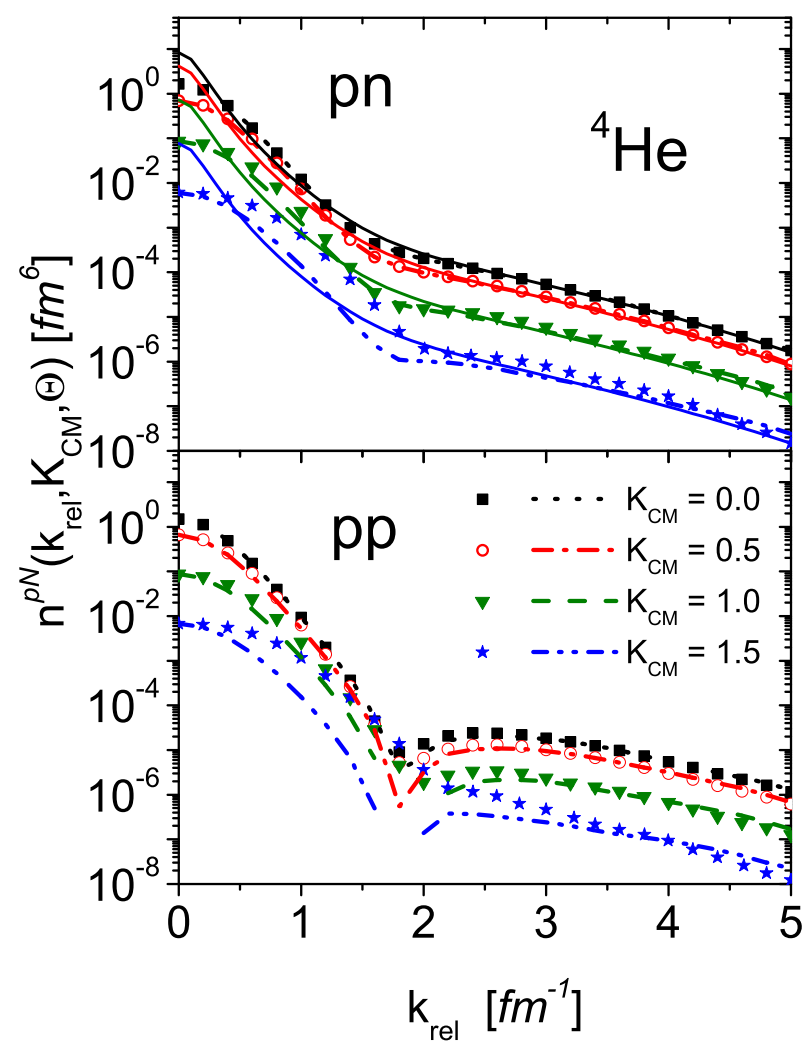

FIG. 2: The same as in Fig. 11 but for ${ }^{4} \mathrm{He}$. Correlated variational wave function from [14] and $A V 8^{\prime}$ interaction [16].

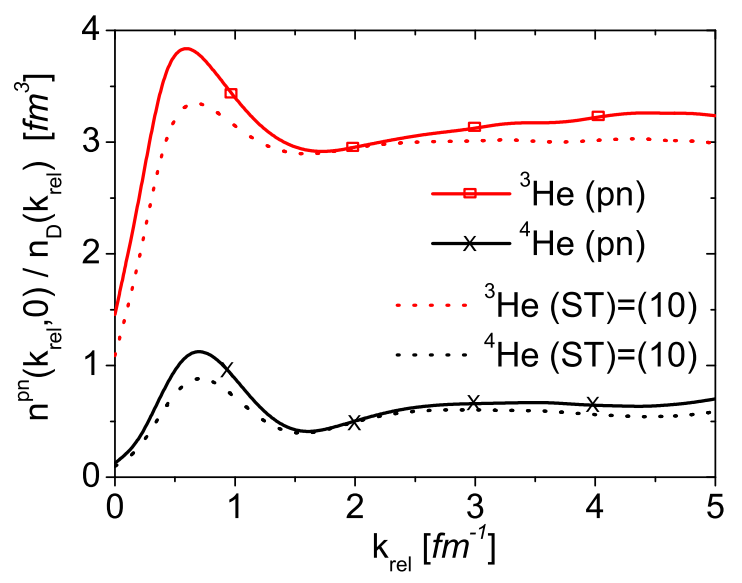

FIG. 3: The ratio of the $p n$ momentum distributions at at $K_{C M}=0$ to the deuteron momentum distributions $n_{D}\left(k_{r e l}\right)$. The contribution from the spin-isospin deuteron states $S=1$, $T=0$ is also shown.

pairs, one $n^{p n}\left(k_{r e l}, K_{C M}, \Theta\right) \simeq n_{D}\left(k_{r e l}\right) n_{C M}^{p n}\left(K_{C M}\right)$, where $n_{D}\left(k_{r e l}\right)$ is the deuteron momentum distribution and the only A-dependence is given by $n_{C M}^{p n / A}\left(K_{C M}\right)$; the factorized form for $p n$ pairs describes the $2 \mathrm{~N}$ SRC config- 


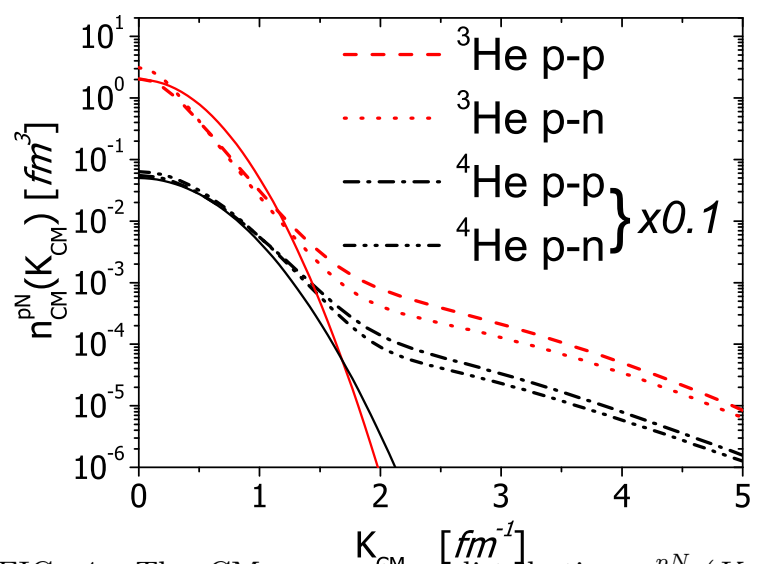

FIG. 4: The CM momentum distribution $n_{C M}^{p N}\left(K_{C M}\right)=$ $\int n^{p N}\left(\boldsymbol{k}_{r e l}, \boldsymbol{K}_{C M}\right) d^{3} k_{r e l}$ for $p p$ and $p n$ pairs in ${ }^{3} \mathrm{He}$ and ${ }^{4} \mathrm{He}$. The solid lines correspond to the model of Ref. [9] aimed at describing the low momentum $\left(K_{C M} \lesssim 1 \mathrm{fm}^{-1}\right)$ part of $n_{C M}^{p N}\left(K_{C M}\right)$.

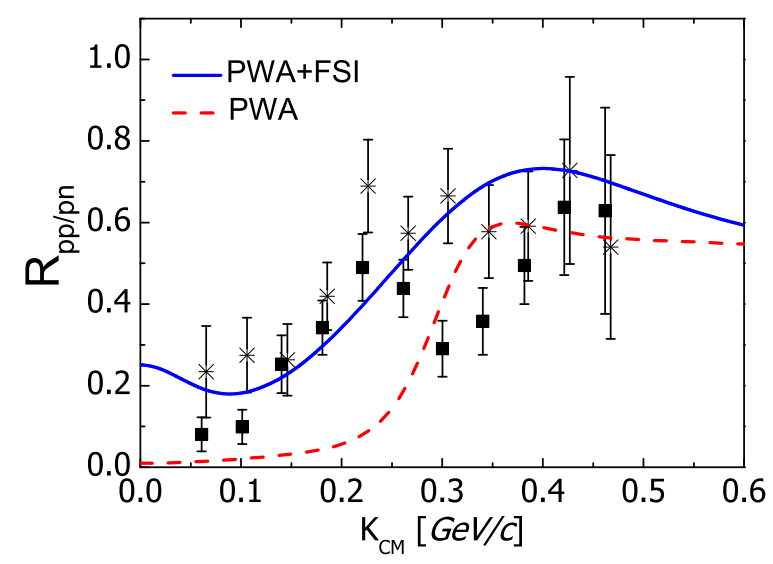

FIG. 5: The ratio of the spectator correlated $p p$ and $p n$ nucleon pairs extracted from the ${ }^{3} \mathrm{He}\left(e, e^{\prime} p p\right) n$ reaction and integrated over the pair relative momentum in the range $1.5<k_{r e l}<3.0 \mathrm{fm}^{-1}$ and the angle $\Theta$ between $\mathbf{K}_{C M}$ and $\mathbf{k}_{\text {rel }}$ [17]. The dashed curve represents the ratio of our calculated momentum distributions and the full curve also includes the effect of the $p p$ and $p n$ rescattering in the final state.

uration, when the relative momentum of the pair is much larger than the CM momentum; (v) at high values of the CM momentum, of the same order of the (large) relative momentum, more than two particles can be locally correlated, with a resulting strong dependence upon the angle and the breaking down of factorization, as clearly shown by Fig. 1 for $K_{C M}=3 \mathrm{fm}^{-1}$. According to our preliminary results [20], all of the above remarks appear to hold also for complex nuclei. Let us now discuss in detail the factorized form of the momentum distributions for $p n$ pairs. To this end we will consider the ratio $R^{p n}=n^{p n}\left(k_{r e l}, 0\right) / n_{D}\left(k_{r e l}\right)$ and its isospin dependence, presented in Fig. 3, and the CM momentum distribution
$n_{C M}^{p N}\left(K_{C M}\right)$, presented in Fig. 4. These two Figures tell us, first of all, that the constant value exhibited by the $S=1, T=0$ ratio at $k_{r e l} \gtrsim 1.5 \mathrm{fm}^{-1}$, is unquestionable evidence that in this region the dependence upon $k_{r e l}$ of the two body momentum distribution $n^{p n}\left(k_{r e l}, 0\right)$ is the same as the deuteron one; secondly, they also tell us that the difference between the ratios for ${ }^{3} \mathrm{He}$ and ${ }^{4} \mathrm{He}$ in the region $k_{r e l} \gtrsim 1.5 \mathrm{fm}^{-1}$ equals exactly the difference between the values of the CM momentum distributions at $K_{C M}=0$, shown in Fig. [4 as a consequence, if we divide the dotted lines by the corresponding values of $n_{C M}^{p n}(0)$, we obtain 1 for both nuclei. Concerning the different behavior of $n_{C M}^{p n}\left(K_{C M}\right)$ for ${ }^{3} \mathrm{He}$ and ${ }^{4} \mathrm{He}$ at $K_{C M} \lesssim 1.5 \mathrm{fm}^{-1}$, this is due to the different binding associated with the CM motion: in ${ }^{3} \mathrm{He}$ the third uncorrelated particle is weakly bound, with a long asymptotic tail, resulting in a sharp peak at $K_{C M}=0$; thus the more rapid fall off of the $\mathrm{CM}$ momentum distributions of ${ }^{3} \mathrm{He}$ leads, with respect to the ${ }^{4} \mathrm{He}$ case, to the wider separation of the curves corresponding to various values of $K_{C M}$ presented in Fig. 1. In ${ }^{4} \mathrm{He}$, the overall average density can already be described by a mean field approach, so that the realistic calculation leads, as shown in Fig. 4, to a result which is practically the same as the one obtained in Ref. 9] within a model based upon the mean value of the kinetic energy in a shell model picture. As for the experimental ratio presented in Fig. 5, it should be pointed out that whereas this quantity represents a nice confirmation of the dominance of tensor correlations, it cannot provide information about the increase or decrease of the two-body momentum distributions with the increase of the CM momentum, since the $p p$ and $p n$ distributions may both increase or decrease at the same time, leaving the ratio almost unchanged. A discriminating quantity would be the ratio of $p n$ (or $p p$ ) pairs in correspondence of two values of the CM momentum. As a matter of fact, it can be seen from Fig. 1 that such a ratio at e.g., $K_{C M}=0$ and $K_{C M}=1.5 \mathrm{fm}^{-1}$, is predicted to be a large positive number.

To sum up, a clear physical picture of the motion of a pair of nucleons embedded in the nuclear medium arises from our calculations. In the region $2 \lesssim k_{\text {rel }} \lesssim 5 \mathrm{fm}^{-1}$, $K_{C M} \lesssim 1 \mathrm{fm}^{-1}$, the motion of $N N$ pairs is governed by $2 \mathrm{~N}$ SRC, characterized by a decoupling of the $\mathrm{CM}$ and relative motions; for a $p n$ pair, the latter is described by the deuteron momentum distribution and the former is governed by the average mean field motion. Some aspects of this picture have already been experimentally confirmed [8], whereas some others, e.g. the CM dependence of two nucleon momentum distributions, need proper experimental investigations. This picture of a locally correlated pair, with the relative motion being practically A-independent, with the A-dependence given only by the CM motion, would be of great usefulness in various fields where SRC correlations have been recently shown to play an important role, such as high energy hadron- 
nucleus [21] and nucleus-nucleus scattering [22], deep inelastic scattering 23], the equation of state of nuclear [24] and neutron [4] matters.

\section{ACKNOWLEDGMENT}

HM and LPK thanks INFN, Sezione di Perugia, for kind hospitality. The Work of MA is supported by the project HadronPhysics2 of the European Commission, grant number 227431. Calculations were performed at CASPUR, thanks to the Standard HPC Grants 2010 SRCNuc and 2011 SRCNuc2.

* On leave from the Bogolubov Lab. Theor. Phys., JINR, 141980 Dubna, Russia, through the program Rientro dei Cervelli of the Italian Ministry of University and Research

[1] S. C. Pieper, R. B. Wiringa, Ann. Rev. Nucl. Part. Sci. 51, 53 (2001).

[2] R. Roth, T. Neff, H. Feldmeier, Prog. Part. Nucl. Phys. 65, 50 (2010).

[3] M. Alvioli, C. Ciofi degli Atti, H. Morita, Phys. Rev. Lett. 100, 162503 (2008).

[4] L. Frankfurt, M. Sargsian, M. Strikman, Int. J. Mod. Phys. A23, 2991(2008).

[5] L. L. Frankfurt, M. I. Strikman, Phys. Rept. 160, 235 (1988).
[6] A. Tang et al, Phys. Rev. Lett. 90, 042301 (2003).

[7] E. Piasetzky et al, Phys. Rev. Lett. 97, 162504 (2006).

[8] R. Subedi et al, Science 320, 1476 (2008).

[9] C. Ciofi degli Atti, S. Simula, Phys. Rev. C53, 1689 (1996).

[10] R. Schiavilla, R. B. Wiringa, S. C. Pieper, J. Carlson, Phys. Rev. Lett. 98, 132501 (2007).

[11] H. Feldmeier, W. Horiuchi, T. Neff, Y. Suzuki, Phys. Rev. C84, 054003 (2011).

[12] R. B. Wiringa, R. Schiavilla, S. C. Pieper, J. Carlson, Phys. Rev. C78, 021001(R) (2008).

[13] A. Kievsky, S. Rosati, M. Viviani, Nucl. Phys. A551, 241 (1993); Private communication.

[14] H. Morita, Y. Akaishi, O. Endo, H. Tanaka, Prog. Theor. Phys. 78, 1117(1987).

[15] R. B. Wiringa, V. G. J. Stoks, R. Schiavilla, Phys. Rev. C51, 38 (1995).

[16] B. S. Pudliner et al, Phys. Rev. C56, 1720 (1997).

[17] H. Baghdasaryan et al [ CLAS Collaboration ], Phys. Rev. Lett. 105, 222501 (2010).

[18] C. Ciofi degli Atti, L. P. Kaptari, S. Scopetta, H. Morita, Few Body Syst. 50, 243(2011).

[19] M. Baldo, M. Borromeo, C. Ciofi degli Atti, Nucl. Phys. A604, 429 (1996).

[20] M. Alvioli, et al to appear.

[21] C. Ciofi degli Atti et al, Phys. Rev. C84, 025205 (2011).

[22] M. Alvioli, M. Strikman, Phys. Rev. C83, 044905 (2011).

[23] E. Piasetzky et al, Nucl. Phys. A855, 245 (2011).

[24] T. Frick, H. Muther, A. Rios, A. Polls, A. Ramos, Phys. Rev. C71, 014313 (2005).

[25] Such an independence has been checked in a wide range of angles 